\title{
Geles de Hidroxipropilcelulosa y Poli(Acrilamida) sintetizados por radiación de microondas (MAOS).
}

\author{
Alfredo Carlos Martínez Arellano, Claudia Alicia Castillo Miranda, Ana Beatriz Morales Cepeda*
}

Centro de Investigación en Petroquímica, TecNM/Instituto Tecnológico de Ciudad Madero, Parque Tecnia (Pequeña y Mediana Industria)Bahía Aldair CP,México.abmoralesc@itcm.edu.mx

\section{Recibido: Noviembre 2017, Aceptado: Diciembre 2017}

\section{Resumen}

La síntesis de geles de hidroxipropilcelulosa / Poliacrilamida mediante radiación de microondas, son una propuesta en la química verde o sustentable, se tiene un tiempo menor de reacción, por espectroscopia de Infrarroja demuestran tener la misma estructura química, la temperatura de transición vítrea se presentan separación de fases. El estudio de hinchamiento del gel en agua, demostró que tiene mayor capacidad de retener agua hasta un 3000 peso\% que su peso inicial, comparado con los geles sintetizados por métodos convencionales se tiene un $10 \%$ más por el método de radiación de microondas.

Palabras clave: Geles, hidroxipropilcelulosa, poli(acrilamida), radiación microondas

\section{Abstract}

The synthesis of hydroxypropylcellulose / polyacrylamide gels by microwave radiation, are a proposal in green or sustainable chemistry, have a lower reaction time, by infrared spectroscopy show to have the same chemical structure, glass transition temperature are presented separation. The study of swelling of the gel in water showed that it has a greater capacity to retain water up to 3000 peso $\%$ than its initial weight, compared with the gels synthesized by conventional methods $10 \%$ more is obtained by the radiation method of microwave.

Key words: Gels, hydroxypropylcellulose, poly (acrylamide), microwave radiation

\section{Introducción}

Nuevas rutas de síntesis de química, surgen de la necesidad de nuevas técnicas rápidas y eficientes, para evitar reacciones de competición existentes en procesos ya conocidos, así como también, eliminar la descomposición de los productos al trabajar en síntesis de fases. En los últimos años, han aparecido muchos informes donde técnicas de preparación convencionales han sido sustituidas por métodos vía microondas $[1,2]$

La síntesis química vía microondas es rápida, simple y energéticamente eficiente. La naturaleza exacta de la interacción de microondas con las especies reaccionantes aún no está clara y es solo especulativa. La transferencia de energía desde el microondas al material se cree que ocurre a través de resonancia o relajación del sistema, lo que repercutiría en un rápido calentamiento del material.

La velocidad de reacción depende de altas temperaturas en métodos de síntesis convencionales o térmicas, esta velocidad de reacción se acelera más fácilmente por medio de síntesis de microondas.
La síntesis por radiación por microondas (MAOS), es una de las técnicas utilizadas como síntesis en la química Verde o sustentable, donde se reduce el consumo de energía, eliminación de residuos y reducción de productos tóxicos $[1,2]$. Los geles de HPC/PAAM han sido sintetizados por método convencional o térmico (3) y ha demostrado ser una opción para la remoción de metales pesados por medio de hinchamiento, también ha demostrado su capacidad para liberación de fármacos [4, 5].

El presente artículo presenta los resultados obtenidos mediante radiación por microondas, en cuanto a su caracterización química, térmica y de hinchamiento, y se abre la oportunidad para estudiar los geles como reservorio en la remoción de metales pesados o liberación de fármacos.

\section{Parte Experimental}


2.1 Reactivos: Acrilamida (AAm). 2-propenamida $\left(\mathrm{C}_{3} \mathrm{H}_{5} \mathrm{NO}\right)$. Marca Aldrich con $97 \%$ de pureza, Hidroxipropil celulosa (HPC). Derivado de la celulosa, marca Aldrich con un peso mol $=10,000 . \mathrm{N}, \mathrm{N}, \mathrm{N}^{\prime}, \mathrm{N}^{\prime}$, Tetrametiletilendiamina (TEMED). 1,2 bi(dimetilamino)etano, $\quad \mathrm{C}_{6} \mathrm{H}_{16} \mathrm{~N}_{2}$ co-catalizador para la formación de radicales libres del APS y hacer la reacción a bajas temperaturas. Marca Sigma-Aldrich con $99 \%$ de pureza, Persulfato de amonio (APS). $\left(\mathrm{NH}_{4}\right)_{2} \mathrm{~S}_{2} \mathrm{O}_{8}$. Iniciador de la polimerización de los acrilatos vía radicales libres. Marca Sigma-Aldrich con 98\% de pureza. Metilénbisacrilamida. (MBAm).N, $\mathrm{N}^{\prime}$, Metilén bis (2-propenamida). $\mathrm{C}_{7} \mathrm{H}_{10} \mathrm{O}_{2} \mathrm{~N}_{2}$. Entrecruzante para la poliacrilamida. Marca Aldrich con una pureza de 99\%, Divinil sulfona (DVS). 1-1'-sulfonilbisetano $\left(\mathrm{C}_{4} \mathrm{H}_{6} \mathrm{O}_{2} \mathrm{~S}\right)$, marca Aldrich con un $97 \%$ de pureza. Entrecruzante específico para la HPC.

2.2 Síntesis de los hidrogeles de HPC/PAAm (Método modificado de Castro Guerrero Carlos Fernando, Phase Behaviour

Hydroxypropylcellulose/Polyacrylamidegels,

Macromoleculare symposia, (296), page 429-435 Editorial Wiley-VCH 2009): La solución consistió de un $90 \%$ de agua desionizada y un $10 \%$ de reactivos en la cantidad deseada para trabajar. Para empezar se cargó 20 $\mathrm{ml}$ de agua desionizada al tubo de ensayo y se agregó $1 \mathrm{~g}$ de HPC, se dejó en agitación alrededor de 15 horas para lograr una solución homogénea, esto a temperatura ambiente. Pasado este tiempo se hizo un barrido con nitrógeno y se agregó $3 \mathrm{~g}$ de AAm. En un vial con $8 \mathrm{ml}$ de agua desionizada se disolvió $0.06 \mathrm{~g}$ de APS junto con $0.003 \mathrm{~g}$ de MBAm y en otro vial con la misma cantidad de agua se disolvió $0.0742 \mathrm{~g}$ de TEMED, ambos viales se dejaron en agitación magnética por 20 minutos. Se realiza la polimerización bajo radiación microondas, usando un microondas marca Anton Parr con una potencia máxima de $1250 \mathrm{~W}$. El tubo de especial del reactor se introdujo en el microondas donde se realizaron 2 ciclos consecutivos de 20 segundos cada uno dejándose reposar por 60 segundos entre los ciclos lo que es equivalente a los $60{ }^{\circ} \mathrm{C}$ que es a la temperatura que se lleva a cabo la polimerización, una vez bien disueltos se inyectó al tubo de ensayo el primer vial, después se adicionó $0.3 \mathrm{ml}$ de DVS y por último se inyectó el segundo vial con TEMED y con agitación constante, posteriormente se realizan 2 ciclos consecutivos y de igual manera que los anteriores. La reacción se polimeriza instantáneamente, y una vez terminada la reacción se vació la solución en una caja petri o en un cristalizador el hidrogel, se deja secar en reposo a $40^{\circ} \mathrm{C}$ en un horno a vacío durante una semana. Una vez seca la película se hace una serie de lavados con agua desionizada para eliminar el monómero $u$ otras sustancias que no hayan reaccionado y se dejó secando nuevamente. Se variaron las relaciones de $25 / 75 \% \mathrm{~mol}, 50 / 50 \% \mathrm{~mol}$ y la relación $75 / 25 \% \mathrm{~mol}$, en la Tabla 2.1 se resumen las concentraciones de derivados y poliacrilamida.

Tabla 2.1.- Relación de HPC/PAAM.

\begin{tabular}{|c|c|}
\hline $\begin{array}{c}\text { Derivado de celulosa/ } \\
\text { Poliacrilamida }\end{array}$ & $\begin{array}{c}\text { Relación } \\
\text { \% mol }\end{array}$ \\
\hline HPC/(PAAm) & $25 / 75$ \\
\hline HPC/(PAAm) & $50 / 50$ \\
\hline HPC/(PAAm) & $75 / 25$ \\
\hline
\end{tabular}

\subsection{Caracterización}

Espectroscopia de Infrarrojo con transformada de Fourier: Para la identificación de las películas de HPC/PAAm se empleó un espectrómetro de transformadas de Fourier marca Perkin Elmer modelo Spectrum One con el accesorio ATR, con un intervalo de frecuencia de $4000 \square 400 \mathrm{~nm}^{-1}$. Con ésta técnica se espera ver los diferentes grupos característicos de la HPC y del PAAm que conforman a las muestras de microgeles.

Calorimetria diferencial de Barrido (DSC): Micro-DSC, marca TA Instruments, se utilizó $500 \mathrm{mg}$ de muestra con una rampa de calentamiento de $1{ }^{\circ} \mathrm{C} / \mathrm{min}$ en un rango de 10 a $150^{\circ} \mathrm{C}$, para mayor sensibilidad en el análisis.

Pruebas de Hinchamiento [4]: Para las pruebas de caracterización de hinchamiento se utilizó agua desionizada. Primero, se cortaron las películas en trozos pequeños, tomando una película de cada una de las muestras, el peso seco de cada muestra es variable. Este peso se consideró como peso inicial $\left(\mathrm{W}_{0}\right)$. Cada pedazo de gel se introdujo en un vial de vidrio con aproximadamente $5 \mathrm{ml}$ de agua desionizada. Se pesó la muestra cada 15 minutos durante la primera hora, después cada hora por 8 horas donde se llega al equilibrio de hinchamiento y posteriormente durante un periodo de 5 días. La cantidad de agua retenida dentro del hidrogel en el equilibrio, se expresa matemáticamente de la siguiente forma, como se menciona a continuación:

$W_{c}(\%)=\frac{W_{h}-W_{s}}{W_{s}} * 100$

Donde:

$\mathrm{W}_{\mathrm{c}}(\%)$ es el porcentaje de hidratación.

$\mathrm{W}_{\mathrm{h}}$ es el peso de la película después del hinchamiento (húmeda).

$\mathrm{W}_{\mathrm{s}}$ es el peso de la película seca.

\section{Resultados y discusión:}

Los geles sintetizados fueron de color blanco flexibles, dependiendo de las concentraciones del HPC o PAAM. La síntesis de los geles se realizó en 30 minutos por radiación de microondas, una síntesis convencional se realiza en 5 horas.

\subsection{Espectroscopia infrarroja por transformada de Fourier (FTIR).}

Mediante la Espectroscopía FTIR se analizaron cualitativamente los grupos funcionales presentes en los geles y se una comparación del efecto de las variables de experimento en los geles ya sintetizados. En la figura 3.1 
se adjunta el espectro IR del gel HPC/AAm, mediante radiación por microondas. A los $3405 \mathrm{~cm}-1$ aparece un pico ancho y fuerte correspondiente al estiramiento de los grupos $\mathrm{OH}$ de la HPC, los picos correspondientes a los NH de la acrilamida, logran apreciarse alrededor de los $3200 \mathrm{~cm}^{-1}$. A los $2973 \mathrm{~cm}^{-1}$ se presenta el estiramiento asimétrico y simétrico, respectivamente, del $\mathrm{CH}_{2}$ de la silla de celulosa de la HPC, en éste gel los picos de la silla de celulosa están muy bien definidos. El siguiente pico es débil que está a los $1678 \mathrm{~cm}^{-1}$ que se atribuye al estiramiento del carbonilo de la poliacrilamida, en esta muestra aparece con una intensidad muy baja debido a la poca concentración de PAAm en el gel. Le sigue un hombro a los $1608 \mathrm{~cm}^{-1}$ que se debe a la flexión $\mathrm{NH}$ de la poliacrilamida. A los $1455 \mathrm{~cm}^{-1}$ aparece un pico que se atribuye a la deformación $\mathrm{CH}_{2}$, al igual que el $\mathrm{C}=\mathrm{O}$ es muy débil, esto nos indica que su presencia se debe a la PAAm. El siguiente pico está a los $1374 \mathrm{~cm}-1$ y se atribuye a la flexión simétrica fuera de plano del $\mathrm{CH}_{2}$ de la HPC. Por último aparece un pico de intensidad fuerte a los $1072 \mathrm{~cm}^{-1}$ que es debido al estiramiento $\mathrm{C}-\mathrm{O}-\mathrm{C}$ de la $\mathrm{HPC}$, éste último pico es el más fuerte de todo el espectro IR, lo que se debe a la alta concentración de HPC en el gel. Los geles sintetizados presentaron las mismas señales de los grupos funcionales.

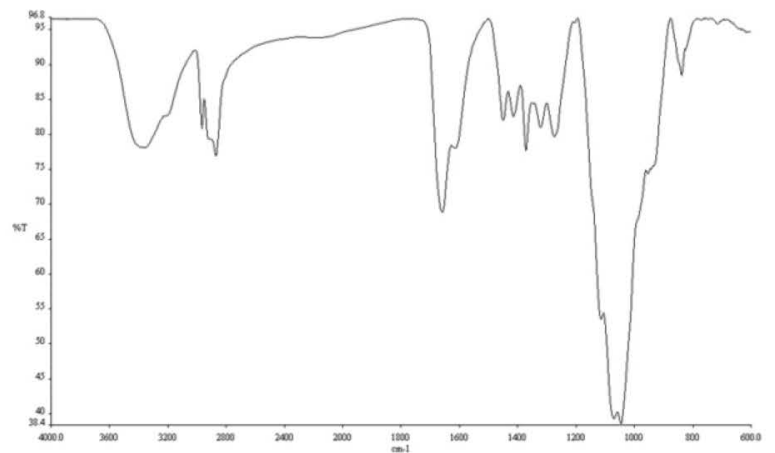

Figura 3.1.- Espectro IR de la muestra HPC/AAm por radiación por microondas.

\section{2- Calorimetría Diferencial de Barrido (DSC).}

El termograma de muestra HPC/PAAm (25/75), mediante radiación por microondas), se muestra en la Figura 3.2, la señal de $\mathrm{T}_{\mathrm{g}}$ a $55^{\circ} \mathrm{C}$, correspondiente a los movimientos laterales de las cadenas de la Hidroxipropilcelulosa (HPC), y la otra señal de $T_{g}$ a $63^{\circ} \mathrm{C}$ es debido a la poliacrilamida (PAAm). La presencia de las dos temperaturas de transición vítrea se tiene una posible separación de fases en el gel, pero la cercanía entre ambas no es posible elucidar esta teoría, aunque el rango de calentamiento fue a $1^{\circ} \mathrm{C} / \mathrm{min}$.

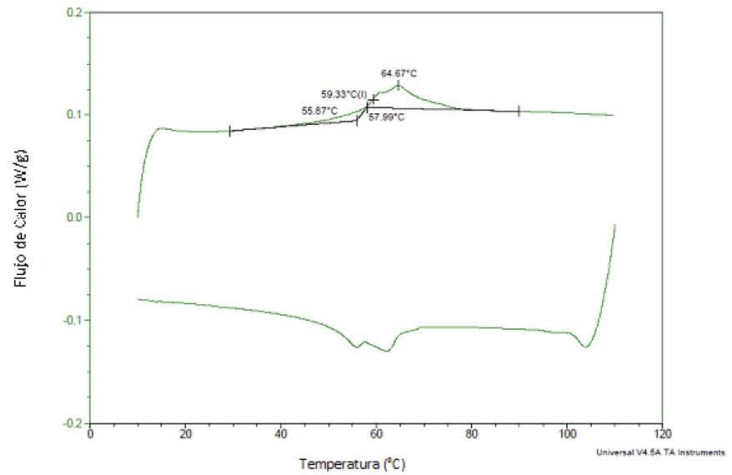

Figura 3.2.- Termograma DSC de la muestra HPC/AAm a 25/75 mediante radiación por microondas.

3.3 Hinchamiento de los geles: El hinchamiento involucra el movimiento de los segmentos del polímero ya que es resultado del incremento en la distancia de separación entre las cadenas del hidrogel.

La figura 3.5, se presenta la gráfica de los porcentajes de hinchamiento de los geles en agua con respecto al tiempo. El hinchamiento de los geles en las tres concentraciones se tiene que las primeras 7 horas es lineal ascendente, posteriormente se tiene un comportamiento lineal con respecto al tiempo hasta las 72 horas ( 3 días), en el caso de los geles con 50 y $25 \%$ de PAAm, se tiene un incremento ascendente en el hinchamiento.

La concentración de 25/75 HPC/PAAm tiene un mayor hinchamiento de agua después de 7 horas en un rango de 3000 peso $\%$ manteniéndose constante hasta 120 horas, al incrementar la concentración de HPC en el gel se observa que el hinchamiento es afectado en escalones donde se tiene que hasta las 72 horas el hinchamiento es de $1420 \mathrm{y}$ $2020 \%$ en peso. Pasadas las 72 horas las cadenas de HPC permiten el incremento de la cantidad de agua en la matriz del gel volviendo a tener la capacidad de retener más cantidad de agua en hasta el doble de lo retenido las primeras 72 horas.

De acuerdo a Castro y col. 2008 [6], sintetizaron los geles de HPC/PAAm, por el método convencional por calentamiento térmico( convección y conducción), logrando que el hinchamiento fuera en un rango de 120 a $330 \%$ en peso, en un tempo de 1500 minutos, por el método de radiación por micro-ondas se tiene 1400 a $3000 \%$ en peso en el mismo tiempo de 1500 minutos. Por lo que se tiene que el arreglo de la red de la red de HPC/PAAm, es más promisoria para las aplicaciones de remoción de metales y liberación prolongada de fármacos [7]. 


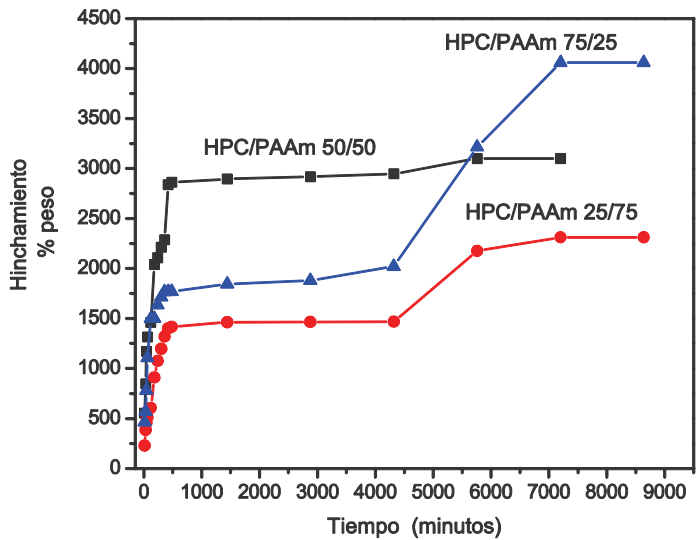

Figura 3.5.- Hinchamiento los geles HPC/AAm sintetizados mediante radiación por microondas en agua.

\section{Conclusiones:}

La síntesis de los geles de Hidroxipropilcelulosa /Poliacrilamida es posible por medio de radiación de microondas (MAOS) reduciendo el tiempo de reacción.

La espectroscopia de Infrarrojo se tiene los mismos grupos funcionales en la síntesis de estos geles por radiación de microondas y métodos convencionales. Separación de fases en el HPC y PAAm en el gel sintetizado por MAOS, lo cual indica que existe una red definida. El hinchamiento de los geles sintetizados por MAOS son $10 \%$ más altos en MAOS que en método convencional.

Este método de síntesis permitirá una aplicación en remoción de metales y liberación prolongada de fármacos.

\section{Agradecimientos:}

Este proyecto fue apoyado por DGEST (TNM) No. 4519.12-13.

\section{Referencias:}

1. Oliver Kappe, Controlled Microwave Heating in Modern Organic Synthesis, Angew. Chem. Int. Ed. 2004, 43, $6250 \square 6284$

2. André Loupy, Ed. Wiley VCH, Microwaves in Organic Synthesis, Germany, 2006

3. C. Castro, A. Morales, A. Koschella, and T. Heinze, $\square$ Phase behaviour of hydroxypropyl cellulose/polyacrylamide $\quad$ gels $\square, \quad$ Macromolecular Symposia, vol. 296, no. 1, 2001, 429 435,

4. Zamarripa Ceron, José Luis; García-Cruz, Juan Carlos; Martínez Arellano, Alfredo Carlos; Castro Guerrero, Carlos Fernando; Angeles San Martin, Marisela Estefanía; Morales $\square$ Cepeda; Ana Beatriz, Heavy metal removal using hydroxypropyl cellulose and polyacrylamide gels, kinetical study, Journal Applied Polymer Science, 2016

5. Claudia Alicia Castillo-Miranda, Ana Beatriz Morales-Cepeda, Carlos Fernando Castro-Guerrero, Homero Salas-Papayanopolos, Hugo Alberto Velasco-
Ocejo, Jorge Alberto González-Sánchez, Ulises ParamoGarcia, and Hugo De Alva-Salazar, Deposition of Ibuprofen Crystals on HydroxypropylCellulose/Polyacrylamide Gel: Experimental and Mathematic Modeling Releasing, , Volume 2016, Article ID 2634104, 8 pages

6. Carlos F. Castro-Guerrero, Ana Morales-Cepeda, José Rivera-Armenta, Ana Mendoza-Martínez, Alberto Álvarez-Castillo Gels from acrylic acid and hydroxypropyl cellulose via free radical polymerization, e-Polymers 2008, no. 147

7. González, N., Prin, J., Benitez, J., Ramírez, A., Garcia, A., Ramírez, M., Sabino, M., Rojas de Gascue, B..

Estudio de la cinética de difusión en hidrogeles sintetizados a partir de acrilamida-co-ácido acrílico con turba y almidón via calentamiento convencional y bajo radiación microondas Revista Latinoamericana de Metalurgia y Materiales, 2012, 32(1): 136-144. 